\title{
A short proof of the odd-girth theorem
}

\author{
E.R. van Dam \\ Dept. Econometrics and O.R. \\ Tilburg University \\ Tilburg, The Netherlands \\ edwin.vandam@uvt.nl
}

\author{
M.A. Fiol \\ Dept. de Matemàtica Aplicada IV \\ Universitat Politècnica de Catalunya \\ Barcelona, Catalonia \\ fiol@ma4.upc.edu
}

Submitted: Apr 27, 2012; Accepted: July 25, 2012; Published: Aug 9, 2012

Mathematics Subject Classifications: 05E30, 05C50

\begin{abstract}
Recently, it has been shown that a connected graph $\Gamma$ with $d+1$ distinct eigenvalues and odd-girth $2 d+1$ is distance-regular. The proof of this result was based on the spectral excess theorem. In this note we present an alternative and more direct proof which does not rely on the spectral excess theorem, but on a known characterization of distance-regular graphs in terms of the predistance polynomial of degree $d$.
\end{abstract}

\section{Introduction}

The spectral excess theorem [10] states that a connected regular graph $\Gamma$ is distanceregular if and only if its spectral excess (a number which can be computed from the spectrum of $\Gamma$ ) equals its average excess (the mean of the numbers of vertices at maximum distance from every vertex), see [5,9] for short proofs. Using this theorem, Van Dam and Haemers [6] proved the below odd-girth theorem for regular graphs.

Odd-girth theorem. A connected graph with $d+1$ distinct eigenvalues and finite oddgirth at least $2 d+1$ is a distance-regular generalized odd graph (that is, a distance-regular graph with diameter $D$ and odd-girth $2 D+1$ ).

In the same paper, the authors posed the problem of deciding whether the regularity condition is necessary or, equivalently, whether or not there are nonregular graphs with $d+1$ distinct eigenvalues and odd girth $2 d+1$. Moreover, they proved this in the negative for the case $d+1=3$, and claimed to have proofs for the cases $d+1 \in\{4,5\}$. In a recent paper, Lee and Weng [14] used a variation of the spectral excess theorem for nonregular graphs to show that, indeed, the regularity condition is not necessary. The odd-girth 
theorem generalizes the result by Huang and Liu [13] that states that every graph with the same spectrum as a generalized odd graph must be such a graph itself. Well-known examples of generalized odd graphs are the odd graphs and the folded cubes.

In this note we give a short and direct proof of the more general result without using any of the spectral excess theorems, but only a known characterization of distance-regularity in terms of the predistance polynomial $p_{d}$ of highest degree.

\section{Preliminaries}

Here we give some basic notation and results on which our proof of the odd-girth theorem is based. For more background on spectra of graphs, distance-regular graphs, and their characterizations, see $[1,2,3,4,7,8]$.

Let $\Gamma$ be a connected graph with vertex set $V$, order $n=|V|$, and adjacency matrix $\boldsymbol{A}$. The spectrum of $\Gamma$ (that is, of $\boldsymbol{A}$ ) is denoted by $\operatorname{sp} \Gamma=\left\{\lambda_{0}^{m_{0}}, \lambda_{1}^{m_{1}}, \ldots, \lambda_{d}^{m_{d}}\right\}$, with distinct eigenvalues $\lambda_{0}>\lambda_{1}>\cdots>\lambda_{d}$, and corresponding multiplicities $m_{i}=m\left(\lambda_{i}\right)$. The predistance polynomials $p_{i}(i=0,1, \ldots, d)$ of $\Gamma$, form a sequence of orthogonal polynomials with respect to the scalar product $\langle f, g\rangle=\frac{1}{n} \sum_{i=0}^{d} m_{i} f\left(\lambda_{i}\right) g\left(\lambda_{i}\right)$, normalized in such a way that $\left\|p_{i}\right\|^{2}=p_{i}\left(\lambda_{0}\right)$. Then, modulo the minimal polynomial of $\boldsymbol{A}$, these polynomials satisfy a three-term recurrence relation of the form

$$
x p_{i}=\beta_{i-1} p_{i-1}+\alpha_{i} p_{i}+\gamma_{i+1} p_{i+1} \quad(i=0,1, \ldots, d),
$$

where we let $\beta_{-1} p_{-1}=0$ and $\gamma_{d+1} p_{d+1}=0$. Note that if $\Gamma$ is distance-regular and $\boldsymbol{A}_{i}$ stands for the distance- $i$ matrix, then $\boldsymbol{A}_{i}=p_{i}(\boldsymbol{A})$ for $i=0,1, \ldots, d$. Our proof of the odd-girth theorem relies mainly on the following result which was first proved in [11] (see also [5], [9]).

Proposition. A connected regular graph $\Gamma$ with $d+1$ distinct eigenvalues is distanceregular if and only if the predistance polynomial $p_{d}$ satisfies $p_{d}(\boldsymbol{A})=\boldsymbol{A}_{d}$.

We remark that it is fairly easy to prove this characterization by backward induction, using the recurrence relation (1), the fact that the Hoffman polynomial $H$ equals $p_{0}+p_{1}+\cdots+p_{d}$, and that for regular graphs $H(\boldsymbol{A})$ equals the all-1 matrix $\boldsymbol{J}$.

By the matrices $\boldsymbol{E}_{i}$ we denote the (principal) idempotents of $\boldsymbol{A}$ representing the orthogonal projections of $\mathbb{R}^{n}$ onto the eigenspaces $\mathcal{E}_{i}=\operatorname{Ker}\left(\boldsymbol{A}-\lambda_{i} \boldsymbol{I}\right)$, for $i=0,1, \ldots, d$. In particular, if $\Gamma$ is regular, then the all-1 vector $\boldsymbol{j}$ is a $\lambda_{0}$-eigenvector and $\boldsymbol{E}_{0}=\frac{1}{n} \boldsymbol{j} \boldsymbol{j}^{\top}=\frac{1}{n} \boldsymbol{J}$. The diagonal entries of these idempotents, $m_{u}\left(\lambda_{i}\right)=\left(\boldsymbol{E}_{i}\right)_{u u}$, have been called the $u$-local multiplicities of the eigenvalue $\lambda_{i}$. Graphs for which these local multiplicities are independent of the vertex $u$ (that is, for which every idempotent has constant diagonal) are called spectrum-regular. The local multiplicities allow us to compute the number of closed 
$\ell$-walks from $u$ to itself in the following way:

$$
a_{u}^{(\ell)}=\left(\boldsymbol{A}^{\ell}\right)_{u u}=\sum_{i=0}^{d} m_{u}\left(\lambda_{i}\right) \lambda_{i}^{\ell} \quad(\ell=0,1,2, \ldots) .
$$

A graph is walk-regular (a concept introduced by Godsil and McKay [12]) if the number

$a_{u}^{(\ell)}$ of closed walks of length $\ell$ does not depend on $u$, for every $\ell=0,1,2, \ldots$ Clearly, a graph is walk-regular if and only if it is spectrum-regular, and every walk-regular graph is regular; properties that will be used in our proof of the odd-girth theorem.

\section{The proof}

Now let us consider a connected graph $\Gamma$ with $d+1$ distinct eigenvalues and finite oddgirth (at least) $2 d+1$, and the corresponding predistance polynomials with recurrence (1). As was shown in [6] by an easy inductive argument, in this particular case we have that $\alpha_{i}=0$ for $i=0,1, \ldots, d-1$ and the polynomials $p_{i}$ are even or odd depending on $i$ being even or odd, respectively. Moreover, $\alpha_{d} \neq 0$ (even though Van Dam and Haemers [6] restrict to regular graphs, the regularity condition is not used by them; the argument is also implicitly used by Lee and Weng [14]). In order to prove the odd-girth theorem, we first need the following lemma.

Lemma. Let $\Gamma$ be a connected graph with $d+1$ distinct eigenvalues and odd-girth $2 d+1$. If $\lambda$ is an eigenvalue of $\Gamma$, then $-\lambda$ is not. In particular, all eigenvalues are nonzero.

Proof. Assume that both $\lambda$ and $-\lambda$ are eigenvalues of $\Gamma$, that is, they are both roots of the minimal polynomial. This means that we can plug in $\lambda$ and $-\lambda$ in the recurrence relations (1) and, in particular, we obtain the two equations $\pm \lambda p_{d}( \pm \lambda)=\beta_{d-1} p_{d-1}( \pm \lambda)+\alpha_{d} p_{d}( \pm \lambda)$. By using that the predistance polynomials are odd or even as indexed, and that $\alpha_{d} \neq 0$, it follows that $p_{d}(\lambda)=0$ (also in the case that $\lambda=0$ ), which is a contradiction (because by the recurrence relations this would imply that $p_{i}(\lambda)=0$ for all $i$, including $i=0$, but $\left.p_{0}=1\right)$.

Now we are ready to prove the general setting of the odd-girth theorem without using the spectral excess theorem.

Theorem. A connected graph $\Gamma$ with $d+1$ distinct eigenvalues and odd-girth $2 d+1$ is distance-regular.

Proof. Fist, let us prove that $\Gamma$ is spectrum-regular (or walk-regular). Since the number of odd cycles with length at most $2 d-1$ is zero we have, using (2),

$$
\sum_{i=1}^{d} m_{u}\left(\lambda_{i}\right) \lambda_{i}^{2 \ell-1}=-m_{u}\left(\lambda_{0}\right) \lambda_{0}^{2 \ell-1} \quad(\ell=1,2, \ldots, d) .
$$


This can be seen as a determined system of $d$ equations and $d$ unknowns $m_{u}\left(\lambda_{i}\right)(i=$ $1,2, \ldots, d)$. Indeed, by the properties of Vandermonde matrices and the above lemma, the determinant of its coefficient matrix is

$$
\begin{aligned}
\left|\begin{array}{cccc}
\lambda_{1} & \lambda_{2} & \cdots & \lambda_{d} \\
\lambda_{1}^{3} & \lambda_{2}^{3} & \cdots & \lambda_{d}^{3} \\
\vdots & \vdots & \ddots & \vdots \\
\lambda_{1}^{2 d-1} & \lambda_{2}^{2 d-1} & \cdots & \lambda_{d}^{2 d-1}
\end{array}\right| & =\prod_{i=1}^{d} \lambda_{i}\left|\begin{array}{cccc}
1 & 1 & \cdots & 1 \\
\lambda_{1}^{2} & \lambda_{2}^{2} & \cdots & \lambda_{d}^{2} \\
\vdots & \vdots & \ddots & \vdots \\
\lambda_{1}^{2 d-2} & \lambda_{2}^{2 d-2} & \cdots & \lambda_{d}^{2 d-2}
\end{array}\right| \\
& =\prod_{i=1}^{d} \lambda_{i} \prod_{d \geqslant i>j \geqslant 1}\left(\lambda_{i}^{2}-\lambda_{j}^{2}\right) \neq 0 .
\end{aligned}
$$

Thus, there exist constants $\alpha_{i}$ such that $m_{u}\left(\lambda_{i}\right)=\alpha_{i} m_{u}\left(\lambda_{0}\right)$, for $i=0,1, \ldots, d$. From this it follows that $m_{u}\left(\lambda_{0}\right) \sum_{j=0}^{d} \alpha_{j}=\sum_{j=0}^{d} m_{u}\left(\lambda_{j}\right)=1$, where the last equality follows from the fact that the sum of all idempotents equals the identity matrix. Thus, for every $i=0,1, \ldots, d, m_{u}\left(\lambda_{i}\right)=\alpha_{i} / \sum_{j=0}^{d} \alpha_{j}$, which does not depend on $u$, and hence $\Gamma$ is spectrum-regular (and walk-regular).

Next, let us show that $p_{d}(\boldsymbol{A})=\boldsymbol{A}_{d}$. Since $\Gamma$ is regular, the Hoffman polynomial $H=$ $p_{0}+p_{1}+\cdots+p_{d}$ satisfies $H(\boldsymbol{A})=\boldsymbol{J}$ and hence $\left(p_{d}(\boldsymbol{A})\right)_{u v}=1$ if $\operatorname{dist}(u, v)=d$. Besides, from the parity of the predistance polynomials, it follows that $\left(p_{i}(\boldsymbol{A})\right)_{u v}=0$ if $\operatorname{dist}(u, v)$ and $i$ have different parity (otherwise, $\Gamma$ would have an odd cycle of length smaller than $2 d+1)$. So $\left(p_{d}(\boldsymbol{A})\right)_{u v}=0$ for every pair of vertices $u, v$ whose distance has a different parity than $d$. If $\operatorname{dist}(u, v)$ is smaller than $d$, but with the same parity, then from the recurrence (1) we get

$$
\left(\boldsymbol{A} p_{d}(\boldsymbol{A})\right)_{u v}=\beta_{d-1}\left(p_{d-1}(\boldsymbol{A})\right)_{u v}+\alpha_{d}\left(p_{d}(\boldsymbol{A})\right)_{u v}=\alpha_{d}\left(p_{d}(\boldsymbol{A})\right)_{u v}
$$

(because $\operatorname{dist}(u, v)$ and $d-1$ have different parity). But the first term is

$$
\sum_{w \in V}(\boldsymbol{A})_{u w}\left(p_{d}(\boldsymbol{A})\right)_{w v}=\sum_{w \in \Gamma(u)}\left(p_{d}(\boldsymbol{A})\right)_{w v}=0
$$

since $\operatorname{dist}(w, v)=\operatorname{dist}(u, v) \pm 1$ has a different parity than $d$. Thus, as $\alpha_{d} \neq 0$, we find that also in this case $\left(p_{d}(\boldsymbol{A})\right)_{u v}=0$. Consequently, $p_{d}(\boldsymbol{A})=\boldsymbol{A}_{d}$ and by the above proposition, $\Gamma$ is distance-regular.

\section{References}

[1] N. Biggs, Algebraic Graph Theory, Cambridge University Press, Cambridge, 1974, second edition, 1993.

[2] A.E. Brouwer, A.M. Cohen, and A. Neumaier, Distance-Regular Graphs, SpringerVerlag, Berlin-New York, 1989. 
[3] A.E. Brouwer and W.H. Haemers, Spectra of Graphs, Springer, 2012; available online at http://homepages.cwi.nl/ aeb/math/ipm/.

[4] D.M. Cvetković, M. Doob, and H. Sachs, Spectra of Graphs, VEB Deutscher Verlag der Wissenschaften, Berlin, second edition, 1982.

[5] E.R. van Dam, The spectral excess theorem for distance-regular graphs: a global (over)view, Electron. J. Combin. 15(1) (2008), \#R129.

[6] E.R. van Dam and W.H. Haemers, An odd characterization of the generalized odd graphs, J. Combin. Theory Ser. B 101 (2011), 486-489.

[7] E.R. van Dam, J.H. Koolen, and H. Tanaka, Distance-regular graphs, manuscript

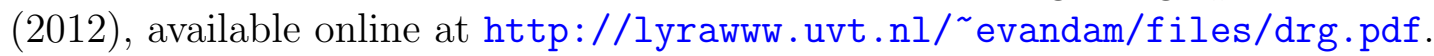

[8] M.A. Fiol, Algebraic characterizations of distance-regular graphs, Discrete Math. 246 (2002), 111-129.

[9] M.A. Fiol, S. Gago, and E. Garriga, A simple proof of the spectral excess theorem for distance-regular graphs, Linear Algebra Appl. 432 (2010), 2418-2422.

[10] M.A. Fiol and E. Garriga, From local adjacency polynomials to locally pseudodistance-regular graphs, J. Combin. Theory Ser. B 71 (1997), 162-183.

[11] M.A. Fiol, E. Garriga, and J.L.A. Yebra, Locally pseudo-distance-regular graphs, J. Combin. Theory Ser. B 68 (1996), 179-205.

[12] C.D. Godsil and B.D. McKay, Feasibility conditions for the existence of walk-regular graphs, Linear Algebra Appl. 30 (1980), 51-61.

[13] T. Huang and C. Liu, Spectral characterization of some generalized odd graphs. Graphs Combin. 15 (1999), 195-209.

[14] G.-S. Lee and C.-w. Weng, The spectral excess theorem for general graphs, J. Combin. Theory Ser. A 119 (2012), 1427-1431. 